\title{
Investigation of Complex Conductivity of Strongly Disordered Superconducting Films by Broadband Flip-Chip Transmission Line Technique
}

\author{
P. Neilinger ${ }^{a, *}$, M. BARÁneK ${ }^{a}$, D. MAnCA $^{a}$ And M. GrajCAR ${ }^{a, b}$ \\ ${ }^{a}$ Department of Experimental Physics, Comenius University, SK-84248 Bratislava, Slovakia \\ ${ }^{b}$ Institute of Physics, Slovak Academy of Sciences, Dúbravská cesta, Bratislava, Slovakia
}

\begin{abstract}
In this paper, we numerically and experimentally test the exploitability of the broadband non-contact flip-chip transmission line technique in studying the complex conductivity of ultra-thin, highly disordered superconducting films of molybdenum carbide. The complex conductivity of such films with various sheet resistances is calculated in the $\mathrm{GHz}$ frequency range by the Mattis-Bardeen model with finite broadening parameter $\Gamma$ and by the Nam model for Dynes superconductors. The transmission of the line in the vicinity of the superconducting thin film is modeled in electromagnetic simulation software, and is compared to experimental data. Evidence of sharp resonances is reported.
\end{abstract}

DOI: 10.12693/APhysPolA.137.797

PACS/topics: disordered superconductor, Dynes superconductor, complex conductivity

\section{Introduction}

Molybdenum carbide (MoC) is a highly disordered superconductor, in which the extent of disorder can be tuned by varying the film thickness. Increased disorder leads to the suppression of superconducting transition temperature $T_{c}$, and to decrease of the Ioffe-Regel product $k_{\mathrm{F}} l$, where $k_{\mathrm{F}}$ is the Fermi wavevector, and $l$ is the mean free path. At critical disorder, when $k_{\mathrm{F}} l \rightarrow 1$, the superconductor-insulator transition occurs. The temperature and frequency dependent complex conductivity $\sigma=\sigma_{1}-\mathrm{i} \sigma_{2}$ of strongly disordered superconducting films deviates from the Mattis-Bardeen (MB) conductivity [1-3], thus indicating a broadened superconducting density of states (SDOS). The broadened SDOS was directly proved by tunneling conductance measurements [4], and analyzed with the Dynes formula

$$
N(E)=\operatorname{Re}\left|\frac{E-\mathrm{i} \Gamma}{\sqrt{(E-\mathrm{i} \Gamma)^{2}-\Delta^{2}}}\right|,
$$

where $\Delta$ is the superconducting energy gap, and $\Gamma$ is the broadening parameter. In order to be able to describe $\sigma$ for $\mathrm{MoC}$ films by measuring a transmission characteristics of resonators fabricated on these films [3], a modification of the MB model [5] with finite $\Gamma$ was introduced [3]. Then, this model was compared to $\sigma$ of Dynes superconductors, derived by Nam's theory [6]. The electromagnetic properties of disordered superconductors are studied in detail in [7].

In this work, we analyze the feasibility of using a broadband non-contact flip-chip transmission line technique

*corresponding author; e-mail: neilinger@fmph.uniba.sk to determine the complex conductivity of $\mathrm{MoC}$ films in the $\mathrm{GHz}$ frequency range. In addition, the special objective was to distinguish between the two models.

\section{Sample preparation and characterisation}

The $\mathrm{MoC}$ films were prepared by magnetron reactive sputtering from a Mo target in an argon-acetylene atmosphere onto c-cut sapphire substrates $5 \times 5 \mathrm{~mm}^{2}$ in size $[8,9]$. The thickness of the sample is set by the sputtering. Decreasing thickness brings about a disorder in film, leading to the suppression of $T_{c}$, and the increase of sheet resistance $R_{s}$. The critical temperature $T_{c}$ rises from $1.3 \mathrm{~K}$ for $t \approx 3 \mathrm{~nm}$ and $R_{s} \approx 980 \Omega$, up to $8.6 \mathrm{~K}$ at $t \approx 30 \mathrm{~nm}\left(R_{s} \approx 65 \Omega\right)$. The suppression of $T_{c}$ in films and the temperature dependence of the normalized $R_{s}$ $\left(R_{s}=210,500,980 \Omega\right)$ are shown in Fig. 1 . The corresponding values $\Gamma$ of the samples, estimated from STS [4], are $\Gamma / \Delta=0.05,0.20$, and 0.4 . For our MoC samples, $\Delta$ is related to $T_{c}$ by the form $\Delta / k_{\mathrm{B}} T_{c}=1.83$.
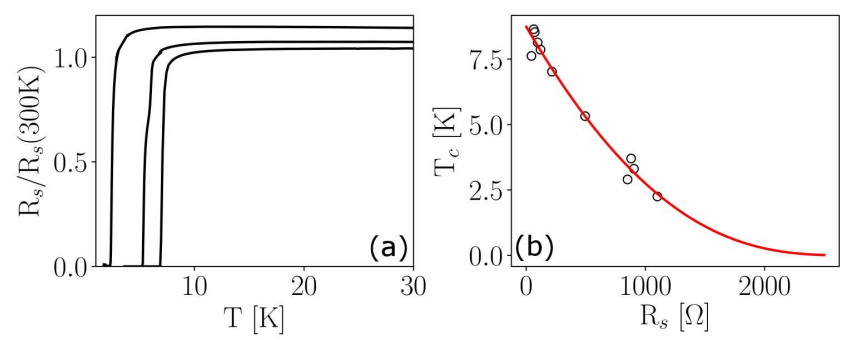

Fig. 1. The normalized $R_{s}$ near $T_{c}$ for MoC films with $R_{s}=210,500,980 \Omega$ (a) and $T_{c}$ as a function of $R_{s}$ for a set of films fitted with Finkelstein's formula [10], showing the suppression of $T_{c}$ with increasing $R_{s}$ (b). 


\section{Complex conductivity}

From the known normal state conductivity $\sigma_{n}=\frac{1}{R_{s} t}$, $T_{c}$ and $\Gamma$, the complex conductivity of Dynes superconductors can be calculated according to the modified MB and the Nam model. However, to be able to distinguish between these two models, as pointed out in [3], either $\mathrm{THz}$ frequency measurements at temperatures well below $T_{c}$ are needed, e.g. [11], or measurements at $\mathrm{GHz}$ frequencies in the vicinity of $T_{c}$ are required. Here, we investigate the second option. The complex conductivity values calculated at $3 \mathrm{GHz}$ (lines), and at $9 \mathrm{GHz}$ (dashed lines), according to the model: MB (circles), Nam (crosses), and modified MB (squares), are shown in Fig. 2 for $R_{s}=210 \Omega(\mathrm{a}, \mathrm{c})$, and $R_{s}=980 \Omega(\mathrm{b}, \mathrm{d})$.
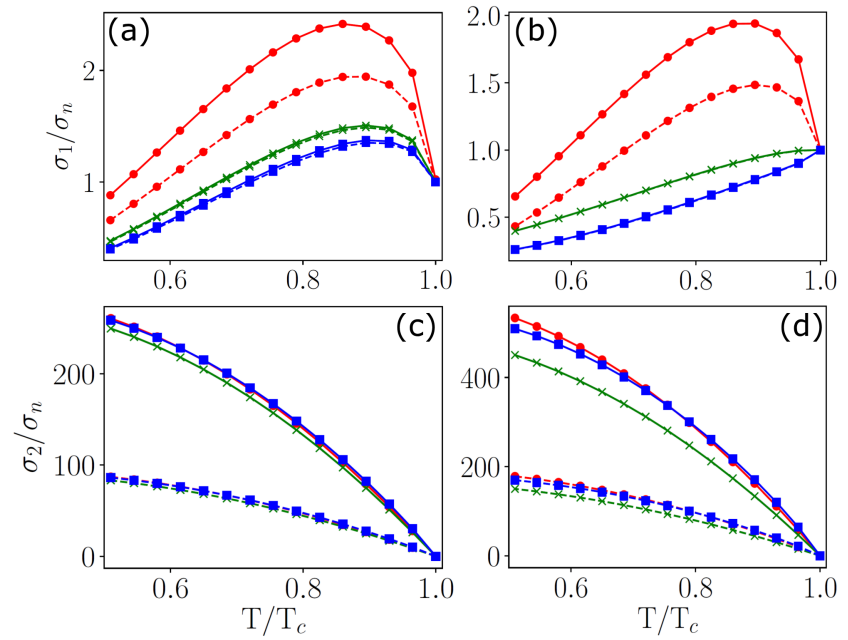

Fig. 2. The real (top) and imaginary (bottom) part of $\sigma$ for a sample with $R_{s}=210 \Omega, \Gamma=0.05 \Delta$ (a,c), and (b,d) $R_{s}=980 \Omega, \Gamma=0.4 \Delta$. The conductivities are calculated at $3 \mathrm{GHz}$ (lines) and $9 \mathrm{GHz}$ (dashed lines) for the MB model (circles), Nam model (crosses) and the modified MB model (squares).

\section{EM Model}

To numerically analyze the response of the transmission line, we modelled a waveguide with a sample positioned across the centerline of the waveguide at $30 \mu \mathrm{m}$ height in SONNET EM software (Fig. 3). The transmission of the line varies with the change of $\sigma$ of the film through the superconducting transmission.

The input to our model is the complex conductivity of the sample as calculated for the studied models. The obtained $S_{21}$ parameters at 3,6 and $9 \mathrm{GHz}$ for the $\mathrm{MB}$ model (circles), Nam model (crosses) and the modified MB model (squares) with $R_{s}=210 \Omega$ and $R_{s}=980 \Omega$ are shown in Fig. 4 a, and b respectively.

The $S_{21}$ parameter of the sample with both low values of $R_{s}$ and $\Gamma$, and at low frequencies increases below $T_{c}$, resembling classical AC susceptibility measurements of superconductors [12]. Note that at higher frequencies (a)

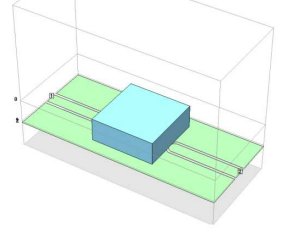

(b)

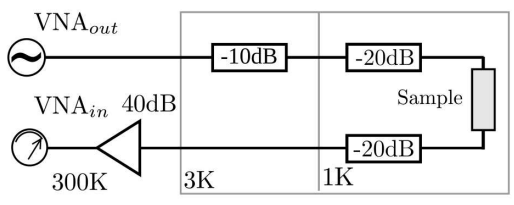

Fig. 3. The 3D model of the coplanar waveguide created in SONNET software. The MoC film is covered with photoresist polymer. The scheme of the measurement set-up (b).

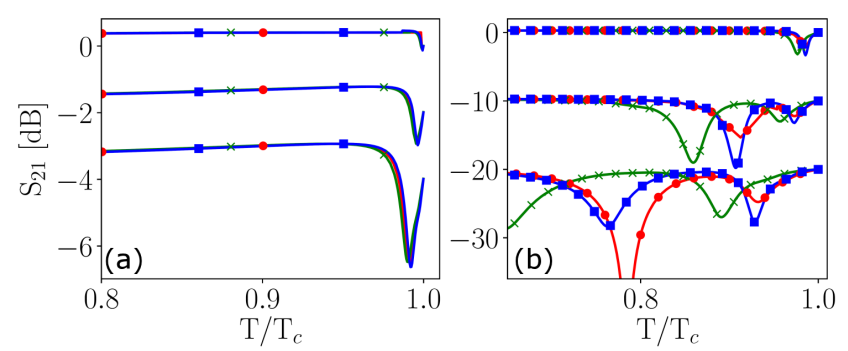

Fig. 4. Temperature dependence of the $S_{21}$ parameter of the transmission line, from top to bottom, at 3,6 and $9 \mathrm{GHz}$ for the MB model (circles), Nam model (crosses) and the modified MB model (squares) for films with $R_{s}=210 \Omega(\mathrm{a})$, and $R_{s}=980 \Omega(\mathrm{b})$. The $S_{21}$ parameters are offset by $-2 \mathrm{~dB}$ and $-10 \mathrm{db}$ for left and right graph, respectively.

a resonance dip occurs before transmission increases. However, the difference between the compared models is negligible. For the highly disordered sample, the temperature dependence at $3 \mathrm{GHz}$ is similar to previous film, but at $6 \mathrm{~Hz}$ and $9 \mathrm{GHz}$ several resonance dips are revealed. The temperature at which these resonances occur is strongly dependent on the complex conductivity model, which enables us to distinguish between the two models.

\section{Experiment}

The coplanar waveguide was milled by means of a circuit board plotter on a Rogers RO4003 PCB. The waveguide was designed to minimalize the reflections and spurious resonances. The temperature dependence measurements were carried out in a He-3 refrigerator.

The transmission was measured by a vector network analyzer, and the signal was amplified by a broadband low noise amplifier. To isolate the sample from room temperature noise, several attenuators were installed on the input and output coaxial lines, see Fig. 3. The calibrated temperature dependence curves of the transmission at 3,6, and $9 \mathrm{GHz}$, are shown in Fig. 5. The transmission of the sample with $R_{s}=210 \Omega$ (Fig. 5a) shows a step-like increase below $T_{c}$. The height of the step increases with frequency, which is consistent with Fig. 4. 

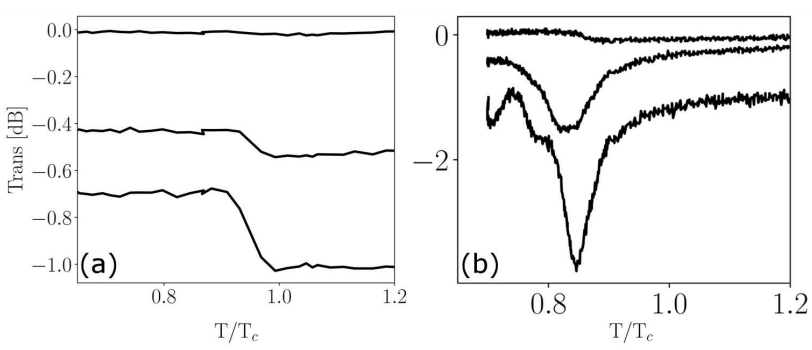

Fig. 5. The measured temperature dependence of the transmission line coupled to the sample with $R_{s}=210 \Omega$ (a) and $R_{s}=980 \Omega(\mathrm{b})$ at 3,6 and $9 \mathrm{GHz}$. The transmission curves are offset by $-2 \mathrm{~dB}$ and $-10 \mathrm{db}$ for left and right graph, respectively.

The transmission for the sample with $R_{s}=980 \Omega$ (Fig. 5b) reveals sizable resonance dips. The intensities of the dips increase with frequency, which which qualitatively agrees with the numerical model.

\section{Conclusions}

We have presented a numerical simulation of the coplanar waveguide's transmission coupled to superconducting $\mathrm{MoC}$ film sputtered on rectangular sapphire substrate. The complex conductivity of the highly disordered superconductor was calculated within the modified MB and the Nam model introduced in [3]. The differences between the temperature dependence of the complex conductivity for the two models manifest in the temperature dependence of the coplanar waveguide's transmission. These differences increase with increased $R_{s}, \Gamma$, and frequency. Due to the high kinetic inductance of the superconducting thin film with high $R_{s}$ and the finite geometry of the sample, including the substrate, the microwave resonances result in a unique transmission pattern. We experimentally proved the presence of these resonances, in qualitative agreement with the numerical simulations. Quantitative analysis of the resonances, which would enable us to distinguish between the suggested models, requires films with well-defined shapes prepared by lithography and their precise positioning above the transmission line. This work is in progress.

\section{Acknowledgments}

This work was supported by the Slovak Research and Development Agency under the contracts APVV16-0068, APVV-16-0372, and APVV-18-0358.

\section{References}

[1] E.F.C. Driessen, P.C.J.J. Coumou, R.R. Tromp, P.J. de Visser, T.M. Klapwijk, Phys. Rev. Lett. 109, 107003 (2012).

[2] P.C.J.J. Coumou, E.F.C. Driessen, J. Bueno, C. Chapelier, T.M. Klapwijk, Phys. Rev. B 88, 180505 (2013).

[3] M. Žemlička, P. Neilinger, M. Trgala et al., Phys. Rev. B 92, 224506 (2015).

[4] P. Szabó, T. Samuely, V. Hašková, J. Kačmarčík, M. Żemlička, M. Grajcar, J.G. Rodrigo, P. Samuely, Phys. Rev. B 93014505 (2016).

[5] D.C. Mattis, J. Bardeen, Phys. Rev. 111, 412 (1958).

[6] Sang Boo Nam, Phys. Rev. 156, 470 (1967).

[7] F. Herman, R. Hlubina, Phys. Rev. B, 96014509 (2017).

[8] M. Trgala, M. Žemlička, P. Neilinger, M. Reh'ak, M. Leporis, Š. Gaži, J. Greguš, T. Plecenik, T. Roch, E. Dobročka, M. Grajcar, Appl. Surf. Sci. 312, 216 (2014).

[9] S.J. Lee, J.B. Ketterson, Phys. Rev. Lett. 64, 3078 (1990).

[10] A.M. Finkel'stein, JETP Lett. 45, 46 (1987).

[11] M. Šindler, C. Kadlec, P. Kužel, K. Ilin, M. Siegel, H. Němec, Phys. Rev. B 97054507 (2018).

[12] M. Nikolo, Am. J. Phys. 63(1), 57 (1995). 